\title{
Sustainable long-term conservation of rare cattle breeds using rotational AI sires
}

\author{
Jean-Jacques ColleAu ${ }^{1 *}$, Laurent AvoN ${ }^{2}$ \\ ${ }^{1}$ INRA, UR337 Station de génétique quantitative et appliquée, \\ 78352 Jouy-en-Josas Cedex, France \\ ${ }^{2}$ Institut de l'élevage, Département Génétique, 149 Rue de Bercy, \\ 75595 Paris Cedex 12, France
}

(Received 21 September 2007; accepted 30 January 2008)

\begin{abstract}
The development of inbreeding in rotation breeding schemes, sequentially using artificial insemination (AI) sires over generations, was investigated for a full AI scheme. Asymptotic prediction formulae of inbreeding coefficients were established when the first rotation list of AI sires (possibly related) was in use. Simulated annealing provided the optimal rotation order of sires within this list, when the sires were related. These methods were also used for subsequent rotation lists, needed by the exhaustion of semen stores for the first bulls. Simulation was carried out starting with groups of independent sires, with different sizes. To generate a yearly inbreeding rate substantially lower than $0.05 \%$ (considered to be within reach by conventional conservation schemes using frequent replacements), the results obtained showed that the number of sires should be at least 10-15 and that the same sires should be used during at least 50 years. The ultimate objective was to examine the relevance of implementing rotation in breeding schemes on the actual rare French cattle breeds under conservation. The best candidate for such a test was the Villard-de-Lans breed (27 bulls and 73000 doses for only 340 females) and it turned out to be the best performer with an inbreeding coefficient of only $7.4 \%$ after 500 years and five different sire lists. Due to the strong requirements on semen stores and on the stability of population size, actual implementation of this kind of conservation scheme was recommended only in special ('niche') cattle populations.
\end{abstract}

conservation / rotation / inbreeding / coancestry / artificial insemination

\section{INTRODUCTION}

Conservation of endangered cattle breeds often involves only several tens or hundreds of individuals. In such circumstances, the prospect of efficient selection for some economically important traits is virtually nil. Then, instead of trying to accumulate genetic gains as in large selected populations, the only

*Corresponding author: ugencjj@dga2.jouy.inra.fr 
appropriate issue to be addressed is to avoid possible genetic losses, first by unfavourable drift and then by inbreeding, the eventual consequence of any drift. A prominent factor of drift is the succession of generations where gene sampling, hence gene losses, continuously occurs. In large selected populations, breeders face (and even underestimate) this risk because they are primarily interested in genetic gains and consequently, replace breeding animals frequently, especially sires. The lifetime of dams can be more or less considered as imposed by the biology of the species. This behaviour still influences breeders of endangered cattle breeds, who are reluctant to use the same sires during very long periods, although semen collection is easy in this species and could provide the stores needed. However, research work has clearly shown that low inbreeding coefficients are definitely possible when rotationally using the same sires during long periods $[11,12,22]$ : the essential reason is that new independent gene samples of the rotation sires are steadily introduced into the population. For instance, when a single non-inbred sire is used throughout over generations, the female gene pool tends towards the gene pool of this sire and then, the inbreeding coefficient tends towards the probability of sampling the same allele in the sire gene pool twice i.e., 0.5 (not 1 , as one might think at first sight).

The objective of this paper will be to examine in detail the asymptotic properties of rotational schemes, using the same rotation list of artificial insemination (AI) sires. The issue of replacing rotation lists, when semen is exhausted, will also be examined, especially to assess the increase, from the current list to the next one, of the average inbreeding coefficients generated. Asymptotic computations will rely on a very simple population structure i.e., discrete generations and no group management (at a given time, the whole female population is born from the same sire). Numerical implementation of the resulting analytical formulae will be carried out in order to assess the value of such an approach to breed conservation under realistic conditions (overlapping generations and asymptotic inbreeding coefficients not exactly obtained) and to identify the major variation factors of the asymptotic inbreeding coefficients.

Finally, very long-term (500 years) deterministic and Monte-Carlo simulations of the rotational AI scheme will be implemented to model some real populations. This time span for evaluating the potential of breeding schemes to contain the development of inbreeding might look excessive and it might be argued that this view is immaterial, given the numerous extraneous risks incurred by rare populations. However, as previously mentioned, these breeds have no other genetic alternative for the long-term future than struggling to keep their genetic background as intact as possible, whatever the level of the other risks. Then, efficient long-term solutions would provide what could be called 
'genetic sustainability'. It should be recognised, however, that good genetic management is not enough to prevent a rare population from disappearing and that strong economic incentives should be found by the corresponding breeders and (or) provided to them.

\section{OUTLINE OF THE APPROACH}

The development pattern of inbreeding in these schemes is stepwise, not continuous, as if levels of inbreeding were represented by the steps of a staircase. The population undergoes each step during a long time unit that might be called the 'interval between step'.

During a given step, a list of $N$ AI sires (generally related) is in use, taking advantage of the semen stores accumulated during the previous step. Simplicity of management for breeders is maximal: based on this list and on the sires of their cows, the breeders involved in the conservation scheme can immediately know which bulls should serve and furthermore, replacement of females can be probabilistic in the sense they are not obliged to replace each female by one daughter, for instance. During this step, inbreeding coefficients go closer and closer to a series of $N$ (generally different) asymptotic values. Section 3 shows how these values can be obtained. The average of these asymptotic values corresponds to the average inbreeding for this step and the $N$ asymptotic values can be considered as cyclic oscillations around this average.

Preparation of semen stores for the next step is compulsory and requires some attention from the staff in charge of the breeding scheme, in contrast with breeders, for whom management is quite simple. Section 4 shows how the next rotation list can be established and how semen stores can be progressively accumulated during the whole step.

From a step to the next one, the average inbreeding coefficient increases, and hence the staircase pattern of inbreeding development, although minor oscillations do exist at a given step of the staircase.

The major requirements for undergoing this kind of breeding scheme are first the initial existence of substantial semen stores, accumulated during the history of the existing conservation breeding scheme, and second the relative stability of the population size.

As mentioned in the introduction, literature on rotation schemes does exist. However, the approach presented here is quite different from the corresponding proposals. The work of Honda et al. [11,12] pertains to selected populations (typically dairy cattle) divided into two tiers, the selection tier subdivided into fully [11] or partly [12] isolated sire lines and the commercial tier, served by 
the selected males, under a rotation scheme. The inbreeding development in the selection tier is of its own, continuous, and does not depend on the genetic situation in the commercial tier. As a result, the inbreeding development in the commercial tier does not follow a staircase pattern but a continuous one mixed with oscillations due to the rotation between lines. We considered that rare populations could not be divided into two distinct tiers due to their small size: in this case, the inbreeding rates in the very small tier procreating AI sires would have been too high. The schemes of Shepherd and Woolliams [22] are quite adapted to small populations but do not consider AI rotation sires beyond generation 2, where generation 0 pertains to the first rotation sires used in history. Here, in contrast, the whole scheme can be conducted forever, theoretically speaking, i.e., unless extraneous events destroy the population.

\section{ASYMPTOTIC PROPERTIES OF A GIVEN ROTATION LIST}

Inbreeding and coancestry issues can always be treated by considering a hypothetical neutral polygenic trait under drift. If its additive genetic variance is 1 , then the coancestry coefficient between two individuals is equal to 0.5 times their covariance for the polygenic trait $[8,15]$. It should be kept in mind that this equivalence is steadily exploited during the subsequent theoretical developments. Furthermore, properties are presented in full matrix notation, avoiding the use of multiple indices (modulo number of sires).

Let us consider an ordered list $\lambda$ of $N$ AI sires: $(1,2, \ldots, N)$. The corresponding vector of breeding values for the neutral polygenic trait is $\mathbf{s}$ with variancecovariance matrix $\mathbf{S}$, i.e., the relationship matrix between the $N$ sires. Let us consider discrete generations and denote $g^{[t]}$ the expected breeding value in the female population at generation $t$, where generation 1 is the initial population. For simplicity, the sire to be used on females of generation $t$ is sire $t(\bmod N)$. At $t=1$, we use sire 1 , at $t=2$, we use sire $2, \ldots$, at $t=N$, we use sire $N$, at $t=N+1$, we use sire 1, and so on. We introduce here the term 'phase': females of phase $i$ are females to be served by sire $i$. Under the rotation regime, females of phase 1 are born from females of phase $N$ and sire $N$. Females of phase $i(i \neq 1)$ are born from females of phase $i-1$ and sire $i-1$.

Let us denote $\mathbf{g}^{[c]}$ the vector of the $N$ successive expected breeding values obtained during cycle $c$ of $N$ generations. Let us introduce the rotation operator matrix $\mathbf{R}$ such that the operation $\mathbf{y}=\mathbf{R x}$ with any column vector $\mathbf{x}$ transforms this vector by transferring the last element to the first place and shifts the other elements i.e., $\mathbf{R}=\left(\begin{array}{cc}\mathbf{0}_{N-1}^{\prime} & 1 \\ \mathbf{I}_{N-1} & \mathbf{0}_{N-1}\end{array}\right)$. To illustrate how this operator mimics 
rotation, let us consider a small example with $N=3$. It can be checked that $y_{1}=x_{3}, y_{2}=x_{1}, y_{3}=x_{2}$. Let us imagine that the $y$ 's pertain to daughters, that the $x$ 's pertain to dams, and that the indices denote the phases defined above, i.e., the sire identification for the next service. It can be checked that the rotation rules were fully followed: daughters of phase 1, phase 2 , phase 3 originated from dams of phases $3,1,2$, respectively, and consequently from sires $3,1,2$, respectively, as required.

Then, returning to the general case and considering vectors of expected breeding values, $\mathbf{g}^{[c+1]}=0.5 \mathbf{R}\left(\mathbf{g}^{[c]}+\mathbf{s}\right)$. It is straightforward that the vector of the expected breeding values found during one cycle will reach the asymptotic value: $\mathbf{g}=0.5\left(\mathbf{I}_{N}-0.5 \mathbf{R}\right)^{-1} \mathbf{R s}=\mathbf{\Omega}$. This means that the asymptotic vector of the $N$ expected breeding values successively obtained during one cycle is a weighted combination of the breeding values of sires. Basically, this equation will allow a fast approach to the calculation of covariances (hence coancestry and inbreeding coefficients) between females and AI sires (see later).

The analytical expression of the terms of weight matrix $\boldsymbol{\Omega}$ can be established according to matrix considerations (see Appendix 1). The following genetic considerations yield the same results in a more accessible way. Let us consider the expected breeding value at generation $N+1$. Then,

$$
g^{[N+1]}=\alpha+\boldsymbol{\beta}^{\prime} \mathbf{r}, \quad \alpha=1 / 2^{N} g^{[1]}, \quad \boldsymbol{\beta}^{\prime}=1 / 2^{N}, \quad 1 / 2^{N-1} \ldots 1 / 2 .
$$

After $k N$ generations (i.e., $k$ cycles), the expected breeding value becomes

$$
g^{[k N+1]}=\alpha 1 / 2^{N(k-1)}+1 / 2^{N(k-1)} \boldsymbol{\beta}^{\prime} \mathbf{s}+1 / 2^{N(k-2)} \boldsymbol{\beta}^{\prime} \mathbf{s}+\ldots+\boldsymbol{\beta}^{\prime} \mathbf{s} .
$$

When $k$ increases, the breeding value tends towards the first element of the asymptotic vector $\mathbf{g}$. Its limit can be obtained from the sum of a geometric series not involving term $\alpha$ and is equal to $\frac{\boldsymbol{\beta}^{\prime} \mathbf{s}}{1-1 / 2^{N}}=\frac{\left(2^{0} \ldots 2^{N-1}\right)}{2^{N}-1} \mathbf{s}$, i.e., $\boldsymbol{\omega}^{\prime} \mathbf{s}$ where $\boldsymbol{\omega}^{\prime}$ represents the first row of matrix $\boldsymbol{\Omega}$. Then, term $\omega_{i}=2^{i-1} /\left(2^{N}-1\right)$. It is straightforward to show that $\sum_{i=1}^{i=N} 2^{i-1}=2^{N}-1$. Then, $\boldsymbol{\omega}^{\prime} \mathbf{1}_{N}=1$ and row 1 of matrix $\boldsymbol{\Omega}$ can be easily interpreted as the relative contribution of the sires' breeding values to the expected breeding value of females of phase 1 . The same reasoning can be carried out for finding the second element of vector $\mathbf{g}$. Then, it turns out that row 2 of matrix $\boldsymbol{\Omega}$ is row 1 after rotation, using the same definition of rotation as previously, but applied to a row vector: $\mathbf{r o w}_{2}=\mathbf{r o w}_{1} \mathbf{R}^{\prime}$. Finally, all the rows can be set up after successive rotations, starting from row 1 . Then, row $i$ of matrix $\boldsymbol{\Omega}$ can be easily interpreted as the relative contribution of the sires' breeding values to the expected breeding value of females of phase $i$. Term $\omega_{i j}$ of matrix $\boldsymbol{\Omega}$ is equal to $\frac{2^{j-i}}{2^{N}-1}$ if $i \leq j$ and to $\frac{2^{N+j-i}}{2^{N}-1}$ otherwise. 


\subsection{Inbreeding of female phases}

The vector of the inbreeding coefficients for the different female phases is vector $\mathbf{F}$. This vector is simple to obtain because it is not influenced by the drift existing on the female population. Due to the probability of sire origins for female phases, the covariance between a female of phase $i$ and her serving sire (sire $i$ ) is the sum of the products between the elements of row $i$ of $\boldsymbol{\Omega}$ and the elements of column $i$ of $\mathbf{S}$. Then, the vector of coancestries between female phases and serving sires is $0.5 \operatorname{Diag}(\mathbf{\Omega S})$. Finally, after introducing the rotation operator, to arrange the result in the appropriate order, we have $\mathbf{F}=0.5 \mathbf{R} \operatorname{Diag}(\mathbf{\Omega S})$. Given the pedigree of AI sires, calculations are straightforward. Therefore, the inbreeding coefficients correspond to a list of $N$ values, possibly different, and show a cyclic pattern, of period $N$, across generations. When sires are unrelated and non-inbred, these values are the same and are equal to $\omega_{11} / 2$, i.e., $1 /\left(2^{N+1}-2\right)$, a value already found by Shepherd and Woolliams [22] and Honda et al. [12]. $\bar{F}$, the average inbreeding coefficient over the cycle, is equal to $\operatorname{Tr}(\mathbf{\Omega S}) / 2 N$ where the symbol Tr refers to the trace of a matrix, i.e., the sum of its diagonal elements. Then, when sires are related, the weight of relationship $s_{i j}$ between sire $i$ and $\operatorname{sire} j(>i)$ is proportional to term $j$ of row $i$ of $\boldsymbol{\Omega}$ plus term $i$ of row $j$ of $\boldsymbol{\Omega}$, i.e., is proportional to the sum $2^{j-i}+2^{N+i-j}$. This expression is minimised when $j-i=N / 2$ or close to $N / 2$, if $N$ is uneven. Then, highly related sires, e.g., sire-son pairs, should be used successively after about half a cycle. More generally, the value of $\bar{F}$ obviously depends on sire order in the rotation list. When $N$ is high, say larger than 10 , it is impossible to test each of the $\overline{N-1}$ ! situations to be envisioned. In this case, Monte-Carlo optimisation methods, such as simulated annealing [14,18,23], can provide an efficient approximate optimum.

\subsection{Coancestries}

For the issue of replacing rotation lists (see further), we need to know coancestry coefficients between female phases and AI males and coancestry coefficients between female phases. The coancestry matrix between female phases (rows) and $\mathrm{AI}$ males (columns) is equal to $0.5 \mathbf{\Omega S}$. The coancestry coefficients between female phases are more complex to obtain: details are given in Appendix 2. These coefficients depend on parameter $\gamma$, the probability that a randomly chosen pair of females is born from different dams.

\section{REPLACEMENT OF ROTATION LISTS}

Semen stores for a given list will be exhausted ultimately. Preparing the next list of AI sires is needed and collection of semen for this list should be 
progressive and anyway fully completed at the time of exhaustion. In the next list, each old sire is represented by a single son, who becomes a new sire, and female phases are represented by a single dam. The mating design for producing the sires of the next list is optimised for minimising the average inbreeding generated by this list.

Replacing lists raises two distinct issues. First, the origin of the new sires, i.e., which is the best list of pairs of parents (AI sire * female phase)? The relationship matrix for a given set of pairs is easy to obtain based on the results given in Section 3.1. Second, semen of replacement sires can only be obtained sequentially due to the small size of the population and due to the need to smooth money expenses over time.

As to the first issue, the problem amounts to finding the best vector of female phases that will be mated to AI sires $1, \ldots, N$. Theoretically $\bar{N}$ ! permutation vectors situations should be tested. Numerical methods such as simulated annealing permit to solve the problem with a reasonable efficiency when factorial becomes too large. For each permutation between the sires attributed to the dams, matrix $\mathbf{S}$ changes and modifies the potential average $\mathrm{F}$ of the progeny born from the corresponding AI list.

To assess the long-term potential of successive rotation lists on simplified populations ( $c f$. later), we further assume that the asymptotic inbreeding coefficients were reached for a given rotation list. Then, there is no difficulty to predict the sets of asymptotic vectors $\mathbf{F}_{\mathbf{1}} \mathbf{F}_{2} \mathbf{F}_{3} \ldots$ corresponding to successive lists $\lambda_{1} \lambda_{2} \lambda_{3} \ldots$ Based on these vectors, we define the value of the inbreeding rate $\Delta F_{i}$ generated by the replacement of list $i-1$ by list $i$ as $\Delta F_{i}=\left(\bar{F}_{i}-\right.$ $\left.\overline{F_{i-1}}\right) /\left(1-\overline{F_{i-1}}\right)$ with setting $\Delta F_{1}=\bar{F}_{1}$. This formula is the same as for conventional $\Delta F$ 's except that the time unit is no longer year or generation but the time interval between steps or equivalently between successive lists.

Replacing lists in real populations raises the second issue i.e., how to proceed sequentially. Further, in these populations, the asymptotic regime might not be obtained at exhaustion of the semen stores due to long intervals between generation and (or) long lists of AI sires.

Hence, here is the procedure we propose for practical populations. First, $\boldsymbol{\varphi}$, the optimal vector of phases of bull dams, is determined ( $\varphi_{i}$ is the phase of the dam mated to AI sire $i$ to produce a replacement son), based on the asymptotic properties of the next list. Then, collection of semen of sons is iterative, sire by sire, starting from a son of sire 1 . When sons of sires $1, \ldots, i-1$ have already been found and collected for semen, the desired son of sire $i$ is obtained from the female of phase $\varphi_{i}$ able to minimise the contributions of the $i$ first sons to the average asymptotic $F$ generated by the new list. This contribution is $\operatorname{Tr}\left(\mathbf{S}_{(i, i)} \mathbf{\Omega}_{(i, i)}\right)$ where block $(i, i)$ of a matrix pertains to its $i$ first rows and the $i$ first 
columns. Finally, after collection of semen for the son of sire $N$, the next $\mathbf{S}$ is fully known and the next asymptotic $\bar{F}$ can be assessed.

The procedures described above for replacing rotation lists have the drawback of inducing high inbreeding coefficients (higher than 10\%) in the replacement sires even as soon as the second or the third list. The algorithm always tries to establish a sort of 'inbred sire lines'. The favourable value of this inbreeding for the long-term inbreeding rate of the overall population has been well known for a long time in population genetics $[5,13,19]$. Partial extra-inbreeding was also proposed for the sake of purging genetic load [3]. However, such high inbreeding rates for the AI sires might impair robustness of the corresponding animals and anyway might have large probability to be rejected by breeders. Then, a dedicated version of simulated annealing was set up in order to contain inbreeding of AI sires close to inbreeding of their female progeny and was tested on simple modelled populations.

\section{PREDICTIONS ON SIMPLE MODELLED POPULATIONS}

In order to assess the potential of rotating breeding schemes, predictions of asymptotic inbreeding coefficients were carried out for six successive lists (a first list constituted of unrelated and non-inbred males, first female generation also constituted of unrelated and non-inbred individuals). Discrete generations and a single herd were assumed, as in the theoretical section.

Different values of $N$ were investigated: 5, 10, 15, and 20. Furthermore, three values were considered for the probability that two randomly chosen females come from different dams: $0.8,0.9$, and 1 . The third value is obtained when each female is replaced by one daughter and the other values correspond to a Poisson distribution of progeny size in a population of 5 and 10 females per generation, respectively. For larger populations and the same distribution of progeny size, the relevant value for the probability considered would have laid between 0.9 and 1 . The assumption of independence between the first AI males did not hold for practical populations. As expected, inbreeding depended not only on the average level of coancestry between sires but also on the full distribution of coancestries. As an example, breed B (see later) with 18 sires exhibiting heterogeneous coancestries around the average $(5.1 \%)$ yielded a $\Delta F_{1}$ of $2.7 \%$. If coancestries had been homogeneous, with the same average, then $\Delta F_{1}$ would have jumped to $5.1 \%$. Then, we thought that presenting a series of results for special configurations might be cumbersome and in fact, little informative. 


\section{SIMULATIONS ON SIX RARE FRENCH CATTLE BREEDS}

The cattle populations under conservation in France and the corresponding breeding schemes are described in [2,7].

For implementing the kind of rotation schemes we described, the current semen stores per bull (National Survey of Year 2005) should be large enough to serve the population in full AI at its current size during 100 years or equivalently twice the population during 50 years (moderate yearly increase of population size: $+2.5 \%$ ), with a minimum number of bulls equal to 8 . In case of an increase of the population, allowance should be made when preparing the semen stores for list 2.

Then, this test retained six breeds: Villard-de-Lans (V, South-East of France), Armoricaine (A, Brittany), Béarnaise (B, South-West), Casta (C, South-West), Froment du Léon (F, Brittany), and Lourdaise (L, South-West). The numbers of cows and heifers of these populations were smaller than 500. The excluded breeds were Bretonne Pie-Noire, Ferrandaise, Maraîchine, Mirandaise, and Nantaise.

\subsection{The current conservation programme of the Villard-de-Lans breed}

The Villard-de-Lans breed is a small population of about 340 cows and heifers, located near Grenoble, southeast of France, exploited both for milk production and beef depending on herds. This breed has been under conservation since 1977 [1]. A substantial effort was devoted to collect semen of bulls as unrelated as possible. Until now, 27 bulls including some sire-son pairs (average coancestry: $3.9 \%$ ) were collected and on an average, 2700 doses were stored per bull (National Survey of Year 2005).

So, this breed was an excellent candidate for implementing a long-term rotation scheme. Monte-Carlo simulations were carried out to investigate the potential of this implementation over a very challenging period (500 years). Furthermore, this scheme was chosen for conducting a comparison between the predicted inbreeding coefficients and the ones observed during simulations.

\subsection{Monte-Carlo AI rotation scenario in the Villard-de-Lans breed}

Although the current natural service (NS) rate is about $40 \%$, we only simulated a full AI scheme (during 500 years, i.e., five lists) for 50 replicates, based on the population structure known at the beginning of 2005 . The sizes of the 49 herds were extremely variable since only eight herds with at least 10 animals 
concentrated $60 \%$ of the animals. Each year, 210 cows and 57 two-year-old heifers were assumed to be inseminated. The average age at culling was 5.4 years and the probability that a breeding female gave birth to a female entering the breeding population was only 0.21 . This probability was kept during simulations. Even when accounting for the large amount of AI not resulting in useful progeny, the existing stores of semen would allow one to inseminate the population during 100 years. Then, this time span was also kept for the subsequent rotation lists. At the beginning, 27 management groups were constituted and mated to each bull of the list. Optimising these groups for progeny inbreeding or allocating them at random was found of little influence on the future development of inbreeding, as expected (data not shown).

Just after starting a rotation list, the best mating programme for producing the bulls of the future list was established. Then, each third year, inseminations by a sire to be replaced were carried out on 10 existing females of the relevant female phase in order to get a son, to be collected for the future list. These females were chosen based on the sequential procedure described previously. Finally, a single son was chosen within the sons born, for mimicking choice of breeders on phenotype.

\subsection{Deterministic predictions for five other rare French cattle breeds}

The value of initiating an AI rotation scheme in these breeds, given the current semen stores and coancestries between sires, was investigated for the same very long period (500 years), using asymptotic equations.

\section{RESULTS}

\subsection{Modelled populations}

Table I shows the results obtained for the AI situation, when 0.9 was the probability of two females randomly chosen originating from different dams. Variation of this parameter by 0.1 around this value modified inbreeding rates between lists by $0.3 \%$, which is small, compared with the range of rates across AI situations (mostly from $2 \%$ to $8 \%$ ). By trial and error, we found that for containing both inbreeding of AI males and inbreeding of their progeny, an efficient simulated annealing procedure should minimise a linear combination of these inbreeding coefficients. The appropriate weights were found to be, respectively, 0.2 and 0.8 . The little weight given to inbreeding of males was enough because male inbreeding varied much more across annealing permutations than female inbreeding. 
Table I. Deterministic predictions on simple AI populations according to the number $N$ of rotation sires.

\begin{tabular}{clllllcc}
\hline$N$ & Step & 1 & 2 & 3 & 4 & 5 & 6 \\
\hline 5 & $\Delta F(\%)$ & 1.61 & 7.46 & 7.46 & 7.51 & 7.68 & 7.72 \\
& $F_{\text {male }}(\%)$ & 0.0 & 3.2 & 13.0 & 20.0 & 22.2 & 29.9 \\
& $F_{\text {female }}(\%)$ & 1.6 & 8.9 & 15.7 & 22.1 & 28.1 & 33.6 \\
10 & $\Delta F(\%)$ & 0.05 & 2.72 & 3.74 & 3.75 & 3.71 & 4.07 \\
& $F_{\text {male }}(\%)$ & 0.0 & 0.5 & 5.0 & 8.1 & 11.9 & 14.5 \\
& $F_{\text {female }}(\%)$ & 0.1 & 2.8 & 6.4 & 9.9 & 13.3 & 16.8 \\
15 & $\Delta F(\%)$ & 0.0 & 1.24 & 2.48 & 2.43 & 2.44 & 2.46 \\
& $F_{\text {male }}(\%)$ & 0.0 & 0.1 & 2.2 & 5.0 & 7.5 & 9.8 \\
& $F_{\text {female }}(\%)$ & 0.0 & 1.2 & 3.7 & 6.0 & 8.3 & 10.6 \\
20 & $\Delta F(\%)$ & 0.0 & 0.65 & 1.88 & 1.73 & 1.88 & 1.80 \\
& $F_{\text {male }}(\%)$ & 0.0 & 0.1 & 1.5 & 3.4 & 5.2 & 7.0 \\
& $F_{\text {female }}(\%)$ & 0.0 & 0.7 & 2.5 & 4.2 & 6.0 & 7.7 \\
\hline
\end{tabular}

For any $N$, the values of $\Delta F_{1}$ obtained with the initial rotation list were low or very low. In contrast, sharp increases were observed with $\Delta F_{2}$ and further increases were observed for subsequent $\Delta F_{i}$ 's but were small after the fourth list. Then, for the very long term $(i \gg 4)$, a reasonable approximation would be $\Delta F_{i} \simeq \Delta F_{4}=\Delta F$. Then, the $\Delta F$ between list was about $7 \%, 4 \%, 3 \%, 1.5 \%$ for $N=5,10,15,20$, respectively, i.e., roughly proportional to $1 / N$. If the same list could be maintained during 50 years, this would give a yearly $\Delta F$ of $0.14 \%$, $0.08 \%, 0.06 \%$ or $0.03 \%$. If the list could be maintained during 100 years, half these values would be obtained. Then, if $N$ was at least $10-15$ and time life of rotation list was at least 50 years, implementing a rotational breeding scheme could compete with conventional conservation breeding schemes, where the lower bound for the yearly $\Delta F$ can be thought of as lying in the vicinity of $0.05 \%$, i.e., $0.30 \%$ per generation in cattle (see later for a discussion of this bound). Table I also shows inbreeding coefficients for males of the list and females resulting from this list. As a result of the procedure used, both inbreeding coefficients were close except for the three first lists where male inbreeding was smaller than female inbreeding.

\subsection{The Villard-de-Lans breed}

In Table II, three sub-periods were sampled from each step: sub-period $a$ (years 1-10), sub-period $b$ (years 46-55), and sub-period $c$ (years 91-100). This sampling allowed one to compare predicted and observed inbreeding coefficients at different moments of the current step and to assess how fast the asymptotic coefficients were obtained. The predicted inbreeding of females born in 
Table II. Observed effect of implementing a rotation breeding scheme in the Villardde-Lans breed.

\begin{tabular}{|c|c|c|c|c|}
\hline \multicolumn{2}{|c|}{$\begin{array}{c}\text { Step of } \\
100 \text { years }\end{array}$} & \multirow{2}{*}{$\begin{array}{c}\begin{array}{c}\text { Observed } \\
\text { inbreeding }\end{array} \\
\text { average } \%(\mathrm{sd} \%) \\
2.0(2.6)\end{array}$} & \multirow{2}{*}{$\begin{array}{c}\text { Phase } \\
\text { inbreeding } \\
(\%)\end{array}$} & \multirow{2}{*}{$\begin{array}{c}\text { Discrepancy } \\
\frac{\sum\left(F_{\text {observed }}-F_{\text {phase }}\right)^{2}}{\sum F_{\text {phase }}^{2}} \\
1.4\end{array}$} \\
\hline 1 & $a$ & & & \\
\hline & $b$ & $1.9(1.0)$ & 1.9 & $10^{-2}$ \\
\hline & $c$ & $1.9(0.9)$ & 1.9 & $5 \times 10^{-5}$ \\
\hline \multirow[t]{3}{*}{2} & $a$ & $3.5(0.7)$ & 3.9 & $4 \times 10^{-2}$ \\
\hline & $b$ & $3.8(0.6)$ & 3.9 & $3 \times 10^{-4}$ \\
\hline & $c$ & $3.9(0.6)$ & 3.9 & $3 \times 10^{-7}$ \\
\hline \multirow[t]{3}{*}{3} & $a$ & $4.8(0.4)$ & 5.2 & $10^{-2}$ \\
\hline & $b$ & $5.1(0.4)$ & 5.2 & $6 \times 10^{-5}$ \\
\hline & $c$ & $5.2(0.4)$ & 5.2 & $3 \times 10^{-7}$ \\
\hline \multirow[t]{3}{*}{4} & $a$ & $6.0(0.4)$ & 6.3 & $5 \times 10^{-3}$ \\
\hline & $b$ & $6.3(0.3)$ & 6.3 & $6 \times 10^{-5}$ \\
\hline & $c$ & $6.3(0.2)$ & 6.3 & $3 \times 10^{-7}$ \\
\hline \multirow[t]{3}{*}{5} & $a$ & $7.0(0.3)$ & 7.4 & $4 \times 10^{-3}$ \\
\hline & $b$ & $7.3(0.2)$ & 7.4 & $6 \times 10^{-5}$ \\
\hline & $c$ & $7.4(0.2)$ & 7.4 & $2 \times 10^{-7}$ \\
\hline
\end{tabular}

Sub-steps $a, b, c$ are years $1-10,46-55,91-100$, respectively.

a given sub-period was obtained from the observed phases and the observed coancestry matrix between males of the current list. At the start of each step, observed inbreeding coefficients were lower than asymptotic coefficients (by about $0.4 \%$ ) except for the first step. In the middle of each step (sub-period $b$ ), asymptotic coefficients were already virtually reached. At the end of each step, discrepancies were very small, thus confirming correctness of the prediction algorithm for the inbreeding produced by a given list. At year 500, inbreeding coefficients were quite reasonable $(7.4 \%)$, corresponding to a very low average yearly $\Delta F(0.007 \%)$ during this extremely long time span, reminding that the current average inbreeding coefficient is $3.9 \%$. In fact, inbreeding decreased during century I and returned to its initial value during century II. Starting from a non-inbred population would also have yielded a very low yearly $\Delta F: 0.015 \%$. This should be compared with the observed yearly value between years of birth 1995 and 2004: $0.08 \%$. Fully deterministic prediction for year 500, as in the simple modelled populations, slightly overestimated inbreeding coefficients. At years 100, 200, 300, 400, and 500, predicted values were 1.9, 4.0, 5.4, 6.6 , and 7.8 whereas observed values were $1.9,3.9,5.2,6.3$, and 7.4 , respectively. A possible explanation was that the actual dams chosen in the beginning 
of periods for generating future sires were less inbred and related to the population than predicted (asymptotic state not reached). Interestingly, standard deviation of the inbreeding coefficients decreased over time. This was in accordance with the decrease of the standard deviation between phases observed during the deterministic prediction concerning the breed. Because this decrease was not observed for simulations concerning initial nuclei of independent sires, this phenomenon could be considered as the result of the progressive dilution of the initial heterogeneity as to inbreeding and coancestry. These interesting perspectives, if applicable, can be compared to the evolution of inbreeding in the Merino Rambouillet flock [17]. After two centuries of closed natural service management (1786-1983), i.e., 49 generations, it climbed up to 52\%, yielding a generational $\Delta F$ of $1.5 \%$ ( $0.04 \%$ per year). This comparison highlights the value of AI and quantitative genetics for conservation issues, largely beyond reach for this flock, due to their availability only after the 1950s.

Sanchez et al. [21] proposed a significant improvement of management methods for 'conventional' (not using rotation sires) populations, by organising sort of rotational female 'phases'. In their method, the number of phases was equal to $N=1+n$ (females) $/ n$ (males). The phases were determined sequentially. Phase 1 produced replacement sons, phase $N$ produced two daughters, and the other ones, one daughter. This scheme would be demanding for breeders, obliged to programme their female replacements very tightly. However, implementing this scheme in the Villard-de-Lans breed would yield a generational $\Delta F$ of $0.3 \%$, i.e., a yearly $\Delta F$ of about $0.05 \%$, at least three times as high as the one found when running the rotation scheme during 500 years (lower than $0.015 \%$, see above). Then, the value of implementing the rotational breeding scheme in this breed was confirmed, provided of course prerequisites would be fulfilled in practice.

\subsection{The five other breeds}

Table III shows the results obtained with these breeds. First, it can be observed that the current yearly inbreeding rate is still substantially larger than the value of $0.05 \%$ we defined as fully satisfactory for a conventional breeding scheme (variation between 0.08 and 0.57 ). The number of rotation sires varied between 8 and 18 and initial coancestry between sires varied between $2.9 \%$ and $7.1 \%$. The values of the eventual inbreeding coefficient at the end of the use of the first list $\left(\Delta F_{1}\right)$ were substantially below $5 \%$ (the limit of acceptability, corresponding to $0.05 \%$ per year during 100 years) for all breeds except for breed L (5.1). However, the long-term increase rate of inbreeding between lists (monitored by $\Delta F_{4}$ ) was rather high (4.8\%) for breeds $\mathrm{A}$ and $F$ with short lists 
Table III. Deterministic prediction of the effect of implementing a rotation breeding scheme in five breeds.

\begin{tabular}{cccccccc}
\hline Breed & Population size & $\Delta F_{y}(\%)$ & List size & Initial sire coancestry $(\%)$ & $\Delta F_{1}(\%)$ & $\Delta F_{4}(\%)$ & $F_{500}(\%)$ \\
\hline A & 136 & 0.08 & 8 & 3.5 & 2.3 & 4.8 & 19.9 \\
B & 149 & 0.25 & 18 & 5.1 & 2.7 & 2.0 & 11.5 \\
C & 206 & 0.47 & 18 & 2.9 & 1.3 & 2.1 & 9.9 \\
F & 232 & 0.57 & 8 & 3.9 & 2.5 & 4.8 & 20.2 \\
L & 245 & 0.33 & 12 & 7.1 & 5.1 & 3.1 & 17.0 \\
\hline
\end{tabular}

$\Delta F_{y}$ : observed yearly inbreeding rate (1995-2004).

$\Delta F_{1}, \Delta F_{4}$ : inbreeding rate calculated for lists 1,4 .

$F_{500}$ : inbreeding of females born at year 500 . 
(eight sires). As a consequence, only breeds $\mathrm{B}$ and $\mathrm{C}$ exhibited an attractive inbreeding coefficient at year 500, 11.5\% and 9.9\%, respectively. However, if conventional breeding schemes would not be able to sustain a yearly inbreeding rate of $0.05 \%$, then initiating rotation breeding schemes in all of the five breeds would still be valuable.

\section{DISCUSSION AND CONCLUSION}

For assessing the relevance of a long-term rotational breeding scheme, we considered that it should perform definitely better than the best of non-rotational (to our sense) schemes, corresponding to the lower bound for $\Delta F$ of about $0.05 \%$ per year. Justification of this value is as follows. Lower bounds for their schemes were indicated by Sanchez et al. [21] to be approximately equal to $1 / 12$ number of males per generation, which corresponds exactly to $0.05 \%$ per year for the Villard-de-Lans breed. As mentioned in [4,10,16,20], an efficient system for managing genetic variability consists in thinking definitely the other way round, as compared with the approach of the present paper, i.e., to replace males very frequently so that the annual number of new males is maximised and also organising circulation of male groups over female groups (for AI or natural service). In these conditions, a generational $\Delta F$ of around $0.3 \%$ might be obtained (i.e., per year, $0.05 \%$ for cattle and $0.07 \%$ for sheep and goats).

This standard of evaluation is far more stringent than the FAO's guidelines [9] that recommend only a generational $\Delta F$ of $1 \%$. Considering both the need for strong long-term sustainability and the major advances obtained in quantitative genetics concerning the design of conservation schemes (see the literature mentioned), a value of $0.5 \%$ would have been much more appropriate and stimulating.

Based on the investigations on theoretical and real cattle populations, one can conclude that rotation breeding schemes might be recommended for implementation in special circumstances, defining sort of population niches. First, census sizes of populations should be stable or exhibiting a moderate increase (order of magnitude: $+2-3 \%$ per year). In France, this excludes breeds exhibiting a sharp revival such as the Ferrandaise, Bretonne Pie-Noire, Maraîchine, and Nantaise breeds. Second, breeders should be already happy with the current sire lines and should envision their further use without any fear. In France, this excludes the Bretonne Pie-Noire breed again, where a strong demand exists since the beginning of the conservation programme (1976) for permanently changing AI sires [6], exactly as in large selected dairy cattle breeds. Outside of the niches where rotation schemes can be used, the schemes proposed by Sanchez et al. [21], provide excellent solutions. 
The possible systematic use of natural service through sons of AI rotation sires was also studied and the corresponding theory fully developed, mainly for a possible implementation in sheep and goats. Theory and results are not shown here, first due to the poor potential assessed on modelled populations and second due to the theoretical complexity.

\section{REFERENCES}

[1] Audiot A., Races d'hier pour l'élevage de demain, INRA éditions, Paris, 1995.

[2] Avon L., Colleau J.J., Conservation in situ de 11 races françaises à très faibles effectifs : bilan génétique et perspectives, Renc. Rech. Rum. 14 (2006) 247250.

[3] Caballero A., Santiago E., Response to selection from new mutation and effective size of partially inbred populations, Genet. Res. Camb. 66 (1995) $213-$ 225.

[4] Chevalet C., de Rochambeau H., Predicting the genetic drift in small populations, Livest. Prod. Sci. 13 (1985) 207-218.

[5] Cockerham C.C., Avoidance and rate of inbreeding, in: Kojima K. (Ed.), Mathematical Topics in Population Genetics, Springer-Verlag, New York, 1970, pp. 104-127.

[6] Colleau J.J., Quéméré P., Larroque H., Sergent J., Wagner C., Gestion génétique de la race bovine Bretonne Pie-Noire. Bilan et perspectives, INRA Prod. Anim. 15 (2002) 221-230.

[7] Danchin-Burge C., Avon L., Analyse de la variabilité génétique de races bovines à très petits effectifs, après vingt ans d'actions de conservation, Renc. Rech. Rum. 7 (2000) 145-148.

[8] Falconer D.S., Mackay T.F.C., Introduction to Quantitative Genetics, 4th edn., Longman Harlow, UK, 1996.

[9] FAO, Secondary Guidelines for the Management of Small Populations at Risk, FAO Publications, Rome, 1998.

[10] Hill W.G., Effective size of populations with overlapping generations, Theor. Popul. Biol. 3 (1972) 278-289.

[11] Honda T., Nomura T., Mukai F., Reduction of inbreeding in commercial females by rotational mating with several sire lines, Genet. Sel. Evol. 36 (2004) 509526.

[12] Honda T., Nomura T., Mukai F., Prediction of inbreeding in commercial females maintained by rotational mating with partially isolated sire lines, J. Anim. Breed. Genet. 122 (2005) 340-348.

[13] Kimura M., Crow J.F., On the maximum avoidance of inbreeding, Genet. Res. Camb. 4 (1963) 279-288. 
[14] Kirkpatrick S., Gelatt C.D., Vecchi M.P., Optimization by simulated annealing, Science 220 (1983) 671-680.

[15] Ollivier L., Éléments de Génétique Quantitative, 2nd edn., INRA, Paris, 2002.

[16] Ollivier L., James J.W., Predicting the annual size of livestock populations, Genet. Res. Camb. 84 (2004) 41-46.

[17] Prod'Homme P., Lauvergne J.J., The Merino Rambouillet flock in the National sheep Fold in France, Small Ruminant Res. 10 (1993) 303-315.

[18] Robert C., Casella G., Monte-Carlo Statistical Methods, Springer-Verlag, New York, 1999.

[19] Robertson A., The effect of non-random mating within inbred lines on the rate of inbreeding, Genet. Res. Camb. (1964) 164-167.

[20] de Rochambeau H., Chevalet C., Minimisation des coefficients de consanguinité dans les petites populations d'animaux domestiques, Génét. Sél. Évol. 17 (1985) 459-480.

[21] Sanchez L., Bijma P., Woolliams J.A., Minimizing inbreeding by managing genetic contributions across generations, Genetics 164 (2003) 1589-1595.

[22] Shepherd R.K., Woolliams J.A., Minimizing inbreeding in small populations by rotational mating with frozen semen, Genet. Res. Camb. 84 (2004) 87-93.

[23] Sonesson A.K., Meuwissen T.H.E., Mating schemes for optimum contribution selection with constrained rates of inbreeding, Genet. Sel. Evol. 32 (2000) $231-$ 248.

\section{APPENDIX 1: FINDING TERMS OF WEIGHT MATRIX $\Omega$}

Matrix $\boldsymbol{\Omega}=\left(\mathbf{I}_{N}-\mathbf{Q}\right)^{-1} \mathbf{Q}$ where $\mathbf{Q}=0.5 \mathbf{R}$. We also have $\boldsymbol{\Omega}=\left(\mathbf{I}_{N}-\mathbf{Q}\right)^{-1}-\mathbf{I}_{N}$. Let $\mathbf{T}=\mathbf{I}_{N}+\mathbf{Q}+\ldots+\mathbf{Q}^{N-1}$. Then, $\left(\mathbf{I}_{N}-\mathbf{Q}\right) \mathbf{T}=\mathbf{I}_{N}-\mathbf{Q}^{N}=\left(1-2^{-N}\right) \mathbf{I}_{N}$ because $\mathbf{R}^{N}=\mathbf{I}_{N}$. Finally,

$$
\mathbf{\Omega}=\frac{2^{N} \mathbf{T}-\left(2^{N}-1\right) \mathbf{I}_{N}}{2^{N}-1}=\frac{\mathbf{I}_{N}+2^{N}\left(\mathbf{Q}+\ldots+\mathbf{Q}^{N-1}\right)}{2^{N}-1}=\frac{\sum_{k=1}^{k=N} 2^{N-k} \mathbf{R}^{k}}{2^{N}-1} .
$$

Matrix $\mathbf{R}$ has its first row constituted of 0 's except for a single 1 at column $N$. Successive rows can be obtained by successive rotation, where a rotation consists in moving the last element to the first place and moving the other elements to the right. First rows of the successive powers of this matrix can be obtained by successive rotations and the other rows can be obtained by internal rotations. Then, the whole matrix $\boldsymbol{\Omega}$ can be built by successive rotations, starting from its first row $\omega$. It is easy to check that term $i$ of this row is equal to $2^{i-1} /\left(2^{N}-1\right)$. 


\section{APPENDIX 2: COANCESTRIES BETWEEN FEMALE PHASES}

Coancestry coefficients between female phases depend on whether they are considered within-cycle or between-cycle. For instance, within-cycle, phase $N$ is separated from phase 1 by $N-1$ generations whereas between-cycle they are separated by only one generation. Here, we consider within-cycle coancestry coefficients. In contrast with inbreeding coefficients, coancestries between female phases are influenced by the drift occurring in females, independently on $\mathrm{AI}$ sires. Here, we decompose the breeding value of an individual into a rotational component due to the AI rotation males and a residual component. It is straightforward that the matrix of coancestry coefficients between phases due to the rotation is $0.5 \Omega \mathbf{S} \Omega^{\prime}$. Let $\mathbf{v}_{\mathbf{1}}$ be the vector, of size $N$, of within-phase residual variances. Then, $\mathbf{v}_{1}=\mathbf{1}_{N}+\mathbf{F}-\operatorname{Diag}\left(\mathbf{\Omega} \mathbf{\Omega} \mathbf{\Omega}^{\prime}\right)$. Within a given phase, some females can share other ancestral origins than AI sires. Then, a residual covariance between two randomly chosen females of the same phase does exist. Let $\mathbf{v}_{\mathbf{2}}$ be the vector, of size $N$, of the within-phase residual covariances. If we denote $\gamma$ the probability that two different females, randomly chosen, originate from different dams, then $\mathbf{v}_{2}=0.25\left((1-\gamma) \mathbf{R} \mathbf{v}_{1}+\gamma \mathbf{R} \mathbf{v}_{2}\right)$. Hence, $\mathbf{v}_{2}=0.25(1-\gamma) \times$ $\left(\mathbf{I}_{N}-0.25 \gamma \mathbf{R}\right)^{-1} \mathbf{R v}_{1}$.

Let $c$ be the vector of residual covariances between successive phases, i.e., covariance between phase 1 and phase 2 , between phase 2 and phase $3, \ldots$ between phase $N$ and phase 1. Obviously, $\mathbf{c}=0.5\left((1-\gamma) \mathbf{v}_{1}+\gamma \mathbf{v}_{2}\right)$. The still unknown covariances between phase $i$ and phase $j(j>i)$ correspond to the domain where $i=1, \ldots, N-2$ and $j=i+2, \ldots, N$. For a given $i$, covariances can be obtained recursively because the covariance between phase $i$ and phase $j$ is equal to 0.5 times the residual covariance between phase $i$ and phase $j-1$. Finally, the off-diagonal terms of the matrix of coancestry coefficients between females are equal to the corresponding terms of the matrix of rotational coancestry coefficients +0.5 times the corresponding terms of the matrix of residual variances-covariances. The diagonal terms are the terms of $\mathbf{v}_{2}$. This algorithm was checked on simulated data. 